\title{
Compatibilização de Blendas de Poliamida 6/ABS usando os Copolímeros Acrílicos Reativos MMA-GMA e MMA-MA. Parte 1: Comportamento Reológico e Propriedades Mecânicas das Blendas
}

\author{
Edcleide M. Araújo \\ Depto. de Engenharia de Materiais, UFCG \\ Elias Hage Jr. \\ Depto. de Engenharia de Materiais, UFSCar \\ Antônio J. F. Carvalho \\ Instituto de Química de São Carlos, USP
}

\begin{abstract}
Resumo: A poliamida 6 (PA6) é um polímero semicristalino de grande aplicação na engenharia e que apresenta instabilidade no processamento e baixa resistência ao impacto sob entalhe. A incorporação do ABS (acrilonitrila-butadieno-estireno) como modificador de impacto da PA6 pode melhorar estas propriedades. Entretanto, como estes materiais são imiscíveis e incompatíveis, torna-se necessária a incorporação de um terceiro componente que promova a interação dessas duas fases imiscíveis. Este estudo analisa a influência dos copolímeros MMA-GMA (metacrilato de metila-metacrilato de glicidila) e MMA-MA (metacrilato de metila-anidrido maléico) como compatibilizantes de blendas PA6/ABS. Estes copolímeros apresentam miscibilidade com a fase $\mathrm{SAN}$ do $\mathrm{ABS}$, devido à presença do PMMA, e têm também os grupos funcionais éster e anidrido capazes de reagir com os grupos terminais da PA6. As blendas PA6/ABS/MMA-MA apresentaram excelente desempenho sob impacto, permanecendo dúcteis em temperaturas subambiente e super-tenazes na temperatura ambiente.
\end{abstract}

Palavras-chave: Compatibilização, tenacificação, poliamida 6, ABS.

Compatibilization of Polyamide 6/ABS Blends using MMA-GMA and MMA-MA Reactive Acrylic Copolymers. Part 1. Rheological and Mechanical Properties of Blends

\begin{abstract}
Polyamide 6 (PA6) is a semicrystalline polymer suitable to be used in engineering applications with a number of advantages, but its processing instability and relatively low impact strength are limiting aspects. The addition of acrylonitrile-butadiene-styrene (ABS) as an impact modifier to PA6 can improve these properties. However, this blend is immiscible and incompatible, hence the use of an adhesion promoter is necessary to improve the interfacial interaction between the phases. This study focuses on the influence of poly (methyl methacrylate-co-glycidyl methacrylate) (MMA-GMA) and poly(methyl methacrylate-co-maleic anhydride) (MMA-MA) copolymers as compatibilizers for PA6/ABS blends. These copolymers are miscible with SAN phase of ABS due to the presence of PMMA and they have also the ester and anhydride functional groups capable of reacting with polyamide end groups. PA6/ABS/MMA-MA blends showed an excellent impact performance, being super-tough at room temperature and maintaining a good ductility below the room temperature.
\end{abstract}

Keywords: Compatibilization, toughness, polyamide, ABS.

\section{Introdução}

As blendas poliméricas constituem uma alternativa para a obtenção de materiais poliméricos com propriedades que, em geral, não são encontradas em um único material. Estas têm sido extensivamente usadas para a tenacificação de plásticos frágeis. Entretanto, geralmente a mistura polimérica necessita de um compatibilizante para melhorar a adesão interfacial, diminuir a tensão superficial e promover a esta- bilidade morfológica da blenda ${ }^{[1]}$. A poliamida 6 (PA6), muito utilizada em blendas poliméricas, apresenta como principais características: elevada tenacidade acima de sua $\mathrm{Tg}$, boa resistência química em meios orgânicos, baixa viscosidade no estado fundido, boa resistência à abrasão, boas propriedades mecânicas e térmicas e, por isso, está inserida na classe dos plásticos de engenharia. Entretanto, algumas de suas características inerentes de rápida cristalização e alta absorção de umidade favorecem à retração do moldado 
e à instabilidade dimensional. Além disso, sua sensibilidade à trinca e fragilidade em temperaturas sub-ambientes torna seu uso restrito a algumas aplicações ${ }^{[2-6]}$. A PA6 possui funcionalidade inerente, através dos grupos terminais aminas $\left(\mathrm{NH}_{2}\right)$ ou carboxílicos $(\mathrm{COOH})$ e, potencialmente, dos grupos amida $(\mathrm{CONH})$. Polímeros funcionalizados com anidrido maléico têm sido apresentados como compatibilizantes bastante efetivos para blendas com PA6, já que os grupos anidridos são capazes de reagir com os grupos terminais amina da PA6 ${ }^{[4,6,7-12]}$. A facilidade de processamento da PA6 e, principalmente, a modificação da sua resistência ao impacto têm sido alvo de estudos para o desenvolvimento de blendas poliméricas. O ABS, copolímero de acrilonitrila-butadienoestireno, tem elevada resistência ao impacto, mesmo em temperaturas sub-ambientes e custo relativamente baixo. A modificação da PA6 com ABS tem sido bastante investigada devido ao desempenho combinado de cada constituinte polimérico $^{[13-19]}$. O objetivo deste trabalho foi estudar o efeito dos copolímeros poli(metacrilato de metila-co-metacrilato de glicidila) (MMA-GMA) e poli(metacrilato de metila-coanidrido maléico) (MMA-MA) como compatibi-lizantes in situ da blenda PA6/ABS. Foram investigadas as características reológica e mecânica das blendas, com e sem compatibilizante.

\section{Experimental}

A Tabela 1 apresenta os materiais usados neste trabalho. A PA6 (Ultramid B3) foi fornecida pela Basf, na forma de grânulos. O ABS foi fornecido pela Nitriflex S.A., na forma de pó. Os compatibilizantes reativos foram sintetizados em laboratório. O MMA-GMA, nas proporções de: 2,5; 5 e 10\% em peso de GMA, foi obtido a partir da copolimerização em massa do metacrilato de metila (MMA) e o metacrilato de glicidila (GMA), utilizando-se como iniciador, o azobisisbutironitrila (AIBN), e o acrilato de etila (EA) como comonômero auxiliar para impedir a degradação térmica tipo "unzipping" do copolímero ${ }^{[17]}$. Eles foram devidamente pesados, selados e imersos em banho termostatizado a $60^{\circ} \mathrm{C}$, por duas horas. O MMA-MA, nas proporções de: 3, 5, $10 \mathrm{e}$ $20 \%$ em peso de MA, foi obtido a partir dos monômeros MMA, EA e o AIBN, como iniciador, através da polimerização em solução, na presença de DMSO (dimetil sulfóxido) como solvente, a $70{ }^{\circ} \mathrm{C}$, por cinco horas. A síntese e a caracterização dos copolímeros estão descritas detalhadamente na literatura $^{[1]}$.

\section{Preparação das Blendas}

Uma extrusora de rosca dupla B\&P $(\mathrm{L} / \mathrm{D}=25,19 \mathrm{~mm}$ em diâmetro) foi utilizada para realizar as misturas, operando a $230{ }^{\circ} \mathrm{C}$ e $170 \mathrm{rpm}$. Corpos de prova de tração e impacto, conforme normas ASTM D638 e D256, respectivamente, foram moldados por injeção utilizando uma injetora Arburg Allrounder $270 \mathrm{~V}$ operando a $240{ }^{\circ} \mathrm{C}$. Todos os materiais à base de PA6 e ABS, foram secos em estufa a vácuo a $80^{\circ} \mathrm{C}$, por pelo menos $24 \mathrm{~h}$, antes de cada etapa de processamento.

\section{Avaliação da Reatividade dos Copolímeros}

A análise da reatividade in situ dos compatibilizantes MMA-GMA e MMA-MA com a PA6 e o ABS foi realizada em Reômetro de Torque Haake, operando-se a uma temperatura de $240{ }^{\circ} \mathrm{C}$ e $60 \mathrm{rpm}$, por $10 \mathrm{~min}$.

\section{Caracterização Mecânica}

Um equipamento Instron, modelo 5569, a uma velocidade de $50,0 \mathrm{~mm} / \mathrm{min}$, foi utilizado para os ensaios de tração e um equipamento Ceast, modelo Resil 25, para os ensaios de impacto. Os corpos de prova de impacto foram entalhados em entalhador Ceast. A temperatura e a umidade relativa onde as amostras foram condicionadas, por no mínimo $40 \mathrm{~h}$ e, onde foram conduzidos os ensaios foram de $23 \pm 2{ }^{\circ} \mathrm{C}$ e $65 \pm 5 \%$, respectivamente. Pelo menos cinco corpos de pro-

Tabela 1. Materiais usados neste trabalho.

\begin{tabular}{|c|c|c|c|}
\hline Polímero & Composição & Peso Molecular (g/mol) & Torque Haake $^{\mathrm{d}}(\mathrm{Nm})$ \\
\hline Poliamida 6 & $\begin{array}{l}\mathrm{GTA}=43,2 \mu \mathrm{eqg}^{-1} \\
\mathrm{GTC}=51,5 \mu \mathrm{eqg}^{-1}\end{array}$ & $\mathrm{Mn}=21000^{\mathrm{a}}$ & 1,07 \\
\hline $\mathrm{ABS}$ & $\begin{array}{c}50 \% \text { PB e } 25 \% \text { AN } \\
\text { em SAN }\end{array}$ & $\begin{array}{l}\mathrm{Mn}=40000^{\mathrm{b}} \\
\mathrm{Mw}=110000^{\mathrm{b}}\end{array}$ & 10,4 \\
\hline MMA-GMA & $10 \%$ GMA & $\begin{array}{l}\mathrm{Mn}=21600^{\mathrm{c}} \\
\mathrm{Mw}=91300^{\mathrm{c}}\end{array}$ & 0,1 \\
\hline \multirow{4}{*}{ MMA-MA } & $3 \% \mathrm{MA}$ & $\begin{array}{l}\mathrm{Mn}=20900^{c} \\
\mathrm{Mw}=40400^{\mathrm{c}}\end{array}$ & 0,01 \\
\hline & $5 \% \mathrm{MA}$ & $\begin{array}{l}\mathrm{Mn}=15800^{\mathrm{c}} \\
\mathrm{Mw}=37900^{\mathrm{c}}\end{array}$ & 0,01 \\
\hline & $10 \% \mathrm{MA}$ & $\begin{array}{l}\mathrm{Mn}=13100^{\mathrm{c}} \\
\mathrm{Mw}=26200^{\mathrm{c}}\end{array}$ & 0,01 \\
\hline & $20 \% \mathrm{MA}$ & $\begin{array}{l}\mathrm{Mn}=10000^{\mathrm{c}} \\
\mathrm{Mw}=16600^{\mathrm{c}}\end{array}$ & 0,01 \\
\hline
\end{tabular}

${ }^{a}$ Medido por análise química dos grupos terminais de cadeia.

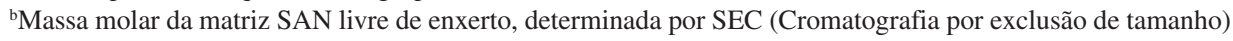

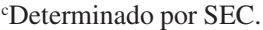

${ }^{\mathrm{d}}$ Torque obtido após $10 \mathrm{~min}$. de corrida. 
va foram utilizados para cada ensaio, que foi realizado na temperatura ambiente e em temperaturas variadas. Para os ensaios em temperaturas variadas, os corpos de prova foram depositados em um forno apropriado acoplado ao aparelho, onde permaneceram, após estabilização da temperatura, por pelo menos 50 min, para temperaturas acima da ambiente, e 30 min, para temperaturas abaixo da ambiente.

\section{Resultados e Discussão}

As Figuras 1 e 2 apresentam as curvas de torque para o copolímero MMA-GMA com PA6 e ABS, respectivamente. Na Figura 1, observa-se um aumento no torque, que significa aumento de viscosidade, o qual só é significativo após aproximadamente 2 min de mistura. Como mencionado por Kudva et al. ${ }^{[17]}$, teores baixos de GMA no copolímero, tais como 2,5 e 5\% em peso, não forneceram bons resultados de resistência ao impacto para as blendas PA6/ABS. Estas apresentaram comportamento frágil na temperatura ambiente, com morfologia grosseira, ou seja, partículas bastante aglomeradas. Enquanto que com 10\% de GMA, os resultados pareceram melhores. Devido a isso, neste trabalho, foi explorada a composição com $10 \%$ de GMA no copolímero. A Figura 2, mostra que a mistura de ABS com o MMA-GMA tem um aumento bastante significativo no torque, o que pode evidenciar reação com o copolímero. Na mistura com $10 \%$

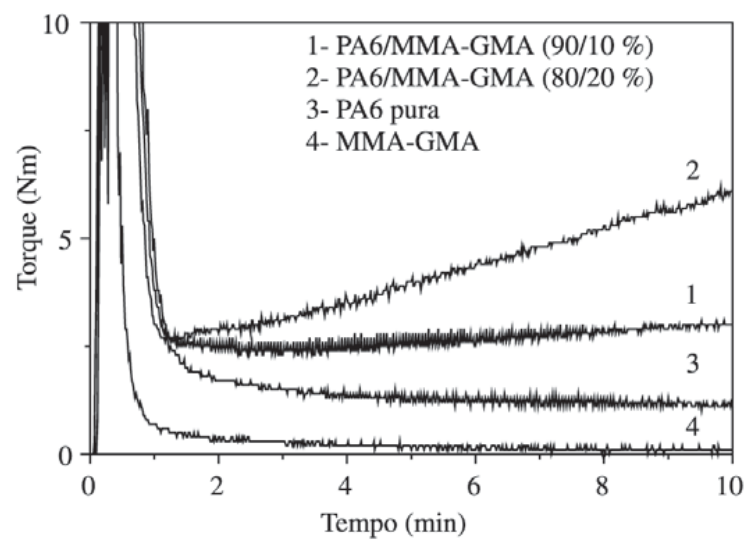

Figura 1. Misturas PA6/MMA-GMA (90/10 e 80/20\%).

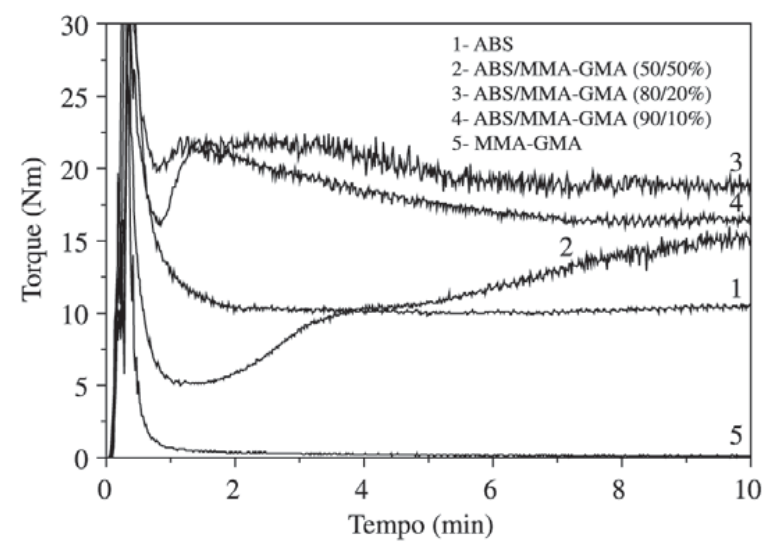

Figura 2. Misturas ABS/MMA-GMA (90/10, 80/20 e 50/50\%). de copolímero, observa-se um aumento significativo do torque, quando comparado ao do ABS e do copolímero puros, indicando elevação de viscosidade, provavelmente devido à reação do grupo epóxi do GMA com o ABS. Acrescendo-se a quantidade de copolímero para $20 \%$, verifica-se um aumento no torque, indicando que a viscosidade da mistura continua aumentando. Entretanto, quando o sistema apresenta $50 \%$ de copolímero, o torque se mantém abaixo daquele das duas misturas anteriormente mencionadas, indicando que, provavelmente, os grupos presentes no ABS disponíveis para reação, já reagiram e o excesso de moléculas de copolímero não tem mais com que reagir e funciona como um diluente, diminuindo a viscosidade da mistura como um todo. Essa reação aparente do ABS com o copolímero pode ser resultado de resíduos de catalisadores e outros aditivos resultantes da síntese do ABS que podem influenciar as reações com epóxis ${ }^{[20]}$, impedindo assim, que o agente compatibilizante permaneça na interface melhorando as propriedades mecânicas sob impacto da blenda, como desejado, o que poderá ser observado mais adiante.

Por outro lado, como mostra a Figura 3, para o copolímero MMA-MA (10\% de copolímero na PA6), tem-se um aumento no torque em tempos relativamente curtos, aproximadamente 1 minuto, que com o aumento do conteúdo de MA no copolímero, continua a elevar-se evidenciando reação de enxertia (graft) dos grupos funcionais da PA6 com o anidrido. O tempo de mistura para que ocorra reação de enxertia (graft) é muito importante, pois deve ser compatível com o tempo de residência na extrusora que foi de $98 \mathrm{~s}$ para a blenda, ou seja, um minuto e meio, que corresponde ao obtido em Reômetro de Torque Haake, necessário para que ocorram reações. Outras composições também foram avaliadas, como 20 e $50 \%$ de copolímero na mistura, entretanto como o número de grupos funcionais amina presentes na PA são limitados, não é necessário adicionar grandes quantidades de copolímero para garantir a reação, pois não existem grupos amina suficientes para tal e, além disso, o copolímero tem viscosidade baixíssima, o que pode diminuir a viscosidade da mistura. Outro fator interessante para esse copolímero é que se pode garantir que não ocorre reação com o ABS, já que o torque se mantém intermediário aos dois polímeros

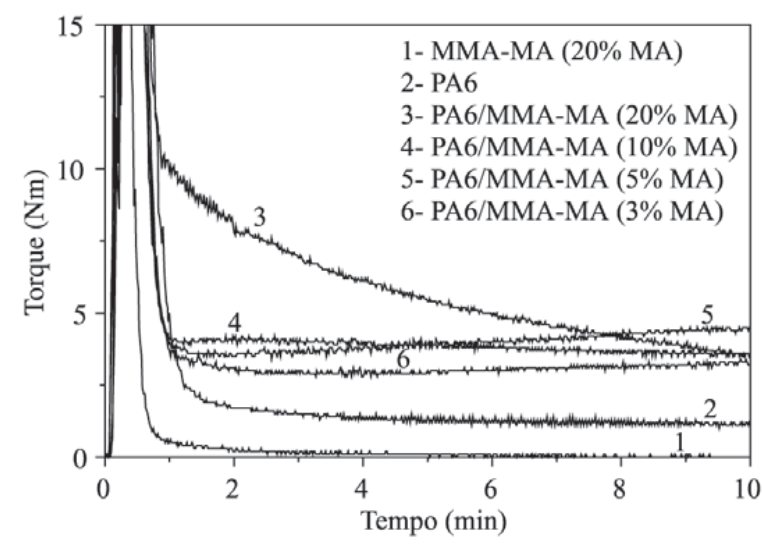

Figura 3. Misturas PA6/MMA-MA (90/10\%). 
puros e diminui quando a quantidade de copolímero elevase, evidenciando apenas um processo de dissolução, como pode ser verificado na Figura 4. Isso pode também assegurar que $\mathrm{o}$ anidrido reage apenas com os grupos terminais amina da PA6, fator decisivo do ponto de vista de compatibilização reativa, ou seja, esse copolímero é ideal para reações de compatibi-lização, já que ele é capaz de reagir apenas com uma fase e ser miscível com a outra, permanecendo na interface entre as duas fases.

A Tabela 2 apresenta as propriedades mecânicas da blenda PA6/ABS compatibilizada com MMA-GMA. A presença de $30 \%$ de ABS na blenda reduz a tensão no escoamento, o módulo de elasticidade e o alongamento na ruptura da matriz. Essa redução é ainda mais acentuada quando se adiciona $50 \%$ de ABS. Como o ABS possui valores de tensão no escoamento e módulo de elasticidade inferiores aos da PA6, tal comportamento era de se esperar parcialmente. Entretanto, a redução significativa do alongamento na ruptura em relação a ambos os polímeros revela a incompatibilidade deste sistema. A adição de $5 \mathrm{pcn}$ (partes por cem de nylon) de copolímero na blenda 70/30\% melhora um pouco a tensão no escoamento e o módulo de elasticidade. Desta forma, alterou-se a sequência de mistura a fim de verificar a influência das possíveis reações que podem ocorrer entre os componentes nas propriedades finais. As seqüências 2, 3 e 4 apresentaram melhores valores de alongamento em relação à 1. Isso pode ser atribuído ao fato de que, como a PA6 tem natu- reza difuncional em relação ao GMA, a dispersão das partículas de ABS é dificultada, provavelmente devido a efeitos de reticulação da PA6, o que pode ter contribuído para valores inferiores no alongamento. Além disso, as prováveis reações entre o emulsificante do ABS e o copolímero, como observado na Figura 2, podem ter desfavorecido a reação dos grupos funcionais da PA6 com o copolímero. Por outro lado, para as outras seqüências $(2,3$ e 4$)$, talvez a reação inicial com o ABS tenha ajudado a dispersar um pouco mais os domínios desta fase na matriz de PA6, permitindo ainda algum tipo de interação com a PA6 e contribuindo para me-

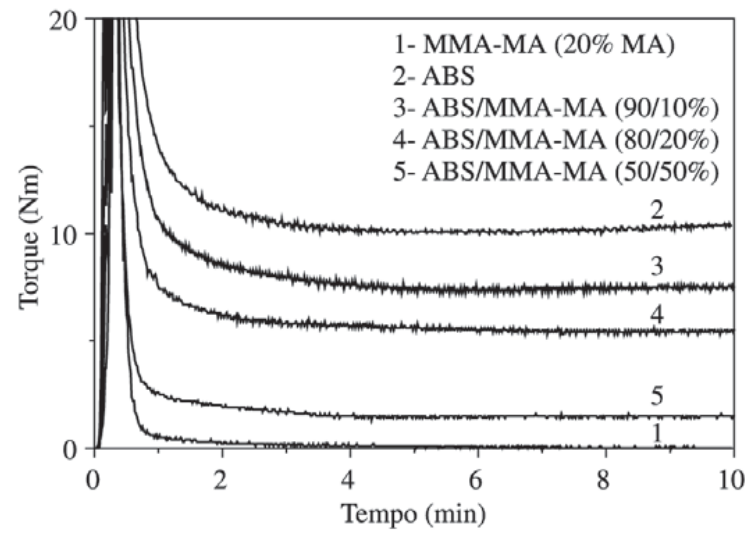

Figura 4. Misturas ABS/MMA-MA (90/10, 80/20 e 50/50\%).

Tabela 2. Propriedades mecânicas da blenda PA6/ABS com o copolímero MMA-GMA (10\% GMA).

\begin{tabular}{|c|c|c|c|c|c|}
\hline Material & $\begin{array}{c}\text { Tensão no } \\
\text { Escoamento (MPa) }\end{array}$ & $\begin{array}{c}\text { Módulo de } \\
\text { Elasticidade (GPa) }\end{array}$ & $\begin{array}{c}\text { Alongamento na } \\
\text { Ruptura (\%) }\end{array}$ & $\begin{array}{c}\text { Resistência ao } \\
\text { Impacto } \operatorname{Izod}(\mathbf{J} / \mathbf{m})\end{array}$ & $\mathbf{T}_{\mathbf{D}-\mathbf{F}}$ \\
\hline PA6 & $72,4 \pm 1,2$ & $2,9 \pm 0,04$ & $87,2 \pm 8,0$ & $31,9 \pm 3,5$ & 69,5 \\
\hline ABS & $14,6 \pm 0,2$ & $1,0 \pm 0,00$ & $181,6 \pm 8,7$ & $396,3 \pm 7,9$ & $-84,0$ \\
\hline $\begin{array}{l}\text { PA6/ABS } \\
(70 / 30 \%)\end{array}$ & $36,6 \pm 1,0^{\mathrm{a}}$ & $1,8 \pm 0,04$ & $65,7 \pm 6,3$ & $69,8 \pm 3,0$ & 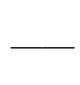 \\
\hline $\begin{array}{l}\text { PA6/ABS/Cop. } \\
\text { (70/30/5 pcn) }\end{array}$ & $45,1 \pm 0,2$ & $2,1 \pm 0,03$ & $50,3 \pm 17,7$ & $191,0 \pm 13,7$ & 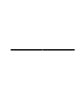 \\
\hline Seqüência 1 & $32,3 \pm 0,7$ & $1,1 \pm 0,04$ & $137,9 \pm 5,8$ & $123,0 \pm 12,4$ & 54,7 \\
\hline Seqüência 2 & $30,3 \pm 2,7$ & $1,1 \pm 0,07$ & $245,1 \pm 4,1$ & $135,2 \pm 14,0$ & 53,3 \\
\hline Seqüência 3 & $32,4 \pm 0,2$ & $1,1 \pm 0,03$ & $260,2 \pm 3,5$ & $108,9 \pm 0,9$ & 65,5 \\
\hline Seqüência 4 & $25,9 \pm 0,5$ & $1,0 \pm 0,02$ & $271,1 \pm 3,9$ & $98,9 \pm 6,4$ & 47,7 \\
\hline $\begin{array}{l}\text { PA6/ABS } \\
(50 / 50 \%)\end{array}$ & $26,7 \pm 0,5$ & $1,4 \pm 0,05$ & $64,9 \pm 6,4$ & $82,2 \pm 2,4$ & - \\
\hline Seqüência 5 & $34,6 \pm 0,7$ & $1,6 \pm 0,06$ & $246,7 \pm 11,6$ & $229,0 \pm 22,4$ & 46,7 \\
\hline Seqüência 6 & $35,8 \pm 0,2$ & $1,7 \pm 0,04$ & $190,0 \pm 8,9$ & $144,5 \pm 11,0$ & 64,3 \\
\hline
\end{tabular}

a Valores calculados com 30\% de inclinação. Para as demais blendas, estes valores foram calculados com inclinação zero.

- pen - partes por cem de nylon.

- Os testes foram realizados em $\mathrm{T}=23 \pm 2{ }^{\circ} \mathrm{C}$ e umidade de $65 \%$.

- Seqüência 1 - PA6/MMA-GMA + ABS $(66,5 / 5+28,5 \%)$.

- Sequiência $2-$ ABS/MMA-GMA + PA6 $(28,6 / 5+66,5 \%)$.

- Sequiência 3 - PA6/ABS + MMA-GMA $(66,5 / 28,5+5 \%)$.

- Sequiência 4 - PA6/MMA-GMA + ABS/MMA-GMA (66,5/2,5 + 28,5/2,5\%).

- Seqüência 5 - PA6/ABS/MMA-GMA (47,5/47,5/5\%).

- Seqüência 6 - ABS/MMA-GMA + PA6 (47,5/5 + 47,5\%).

- Módulo tangente a $0,5 \%$.

- $\mathrm{T}_{\mathrm{D}-\mathrm{F}}-$ Temperatura de transição dúctil-frágil que é o ponto médio, entre os pontos máximo e mínimo, na curva de resistência ao impacto Izod vs. temperatura. 
lhores propriedades. Para efeito de comparação com o trabalho do Kudva et al. ${ }^{[17]}$, foram produzidas blendas de PA6/ABS/MMA-GMA, na proporção 47,5/47,5/5\%, com estes componentes misturados simultaneamente, o que foi denominado, neste trabalho, como sequiência 5. Também foi produzida uma seqüência de mistura, denominada de seqüência 6 , semelhante à seqüência 2 , já que esta apresentou melhor desempenho de impacto que será verificado mais adiante. Esta composição não apresentou diferenças significativas em relação à composição anterior, como verificado na Tabela 2. Observando-se o módulo de elasticidade dessas seqüências, este parece ter tido um aumento em relação às outras seqüências de mistura, mesmo tendo uma maior quantidade de ABS. Como já mencionado, provavelmente um maior conteúdo de ABS favoreça as reações com o copolímero, melhorando a dispersão das partículas e as propriedades.

A influência do copolímero MMA-MA na blenda PA6/ABS foi estudada por meio das propriedades mecânicas. A Tabela 3 mostra as propriedades mecânicas das blendas. Observa-se que o compatibilizante exerce grande influência no valor destas propriedades. Todas elas melhoraram seus valores em relação à blenda sem compatibilizante. Para a composição 47,5/47,5/5\%, 3 \% MA no copolímero já são suficientes para melhorar as propriedades, tendo até, um valor maior na tensão no escoamento e no módulo de elasticidade, em relação às outras concentrações. Isso pode ser atribuído à elevada rigidez deste copolímero em relação aos demais. Em princípio, um aumento na massa molar proporciona um aumento em ambas as propriedades para qualquer sistema polimé-rico ${ }^{[21]}$. Além disso, como verificado nas curvas de reatividade obtidas no reômetro, o copolímero MMAMA é capaz de reagir apenas com os grupamentos amina da PA6 e permanecer miscível com a fase do ABS, garantindo a compatibi-lização da blenda. Diante destes resultados, explorou-se, então, a composição 70/30\%, apenas com os copolímeros com 3 e 5\% MA. Isto porque a influência da concentração de MA no copolímero é pouco significativa para todas as propriedades analisadas. As concentrações utilizadas proporcionam aumentos semelhantes em todas as propriedades.

Oshinski et al. ${ }^{[22]}$ estudaram blendas de PA6 com as borrachas SEBS e EPR. Eles observaram que, em geral, valores melhores de tenacidade são alcançados para massa molar mais alta de poliamida 6 , tanto em temperatura ambiente quanto em baixas temperaturas. Além disso, o tamanho de partícula de borracha também afeta os valores da tenacidade em baixas temperaturas. As Figuras 5 e 6 apresentam as curvas da resis-

Tabela 3. Propriedades mecânicas da blenda PA6/ABS com o copolímero MMA-MA (3, 5, 10 e 20\% MA),

\begin{tabular}{|c|c|c|c|c|c|}
\hline Material & $\begin{array}{c}\text { Tensão no } \\
\text { Escoamento (MPa) }\end{array}$ & $\begin{array}{c}\text { Módulo de } \\
\text { Elasticidade (GPa) }\end{array}$ & $\begin{array}{c}\text { Alongamento na } \\
\text { Ruptura(\%) }\end{array}$ & $\begin{array}{c}\text { Resistência ao } \\
\operatorname{Impacto} \operatorname{Izod}(\mathrm{J} / \mathrm{m})\end{array}$ & $\mathbf{T}_{\text {D-F }}$ \\
\hline PA6 & $72,4 \pm 1,2^{\mathrm{a}}$ & $2,9 \pm 0,04$ & $87,2 \pm 8,0$ & $31,9 \pm 3,5$ & 69,5 \\
\hline ABS & $14,6 \pm 0,2^{\mathrm{a}}$ & $1,0 \pm 0,00$ & $181,6 \pm 8,7$ & $396,3 \pm 7,9$ & $-84,0$ \\
\hline $\begin{array}{l}\text { PA6/ABS } \\
(70 / 30 \%)\end{array}$ & $36,6 \pm 1,0^{\mathrm{b}}$ & $1,8 \pm 0,1$ & $65,7 \pm 6,3$ & $69,8 \pm 3,0$ & $\longrightarrow$ \\
\hline $\begin{array}{l}\text { PA6/ABS/Cop. } \\
(3,0 \% \mathrm{MA})^{\mathrm{c}}\end{array}$ & $43,4 \pm 2,0^{a}$ & $1,9 \pm 0,1$ & $356,0 \pm 29,0$ & $785,8 \pm 24,3$ & 9,4 \\
\hline $\begin{array}{l}\text { PA6/ABS/Cop. } \\
(5,0 \% \text { MA })^{\mathrm{c}}\end{array}$ & $41,0 \pm 1,5^{\mathrm{b}}$ & $2,0 \pm 0,1$ & $292,0 \pm 21,6$ & $810,7 \pm 14,0$ & 9,4 \\
\hline $\begin{array}{l}\text { PA6/ABS/Cop. } \\
(3,0 \% \mathrm{MA})^{\mathrm{d}}\end{array}$ & $38,8 \pm 2,1^{\mathrm{b}}$ & $1,8 \pm 0,1$ & $277,7 \pm 12,3$ & $112,4 \pm 5,0$ & 28,2 \\
\hline $\begin{array}{l}\text { PA6/ABS/Cop. } \\
(5,0 \% \mathrm{MA})^{\mathrm{d}}\end{array}$ & $42,8 \pm 3,6^{\mathrm{a}}$ & $1,9 \pm 0,1$ & $350,9 \pm 34,6$ & $839,1 \pm 22,8$ & 11,2 \\
\hline $\begin{array}{l}\text { PA6/ABS } \\
(50 / 50 \%)\end{array}$ & $26,7 \pm 0,5^{\mathrm{a}}$ & $1,4 \pm 0,1$ & $64,9 \pm 6,4$ & $82,2 \pm 2,4$ & 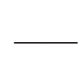 \\
\hline $\begin{array}{l}\text { PA6/ABS/Cop. } \\
(3,0 \% \mathrm{MA})^{\mathrm{e}}\end{array}$ & $33,7 \pm 0,9^{b}$ & $1,5 \pm 0,1$ & $359,5 \pm 23,9$ & $849,9 \pm 3,2$ & $-10,0$ \\
\hline $\begin{array}{l}\text { PA6/ABS/Cop. } \\
(5,0 \% \mathrm{MA})^{\mathrm{e}}\end{array}$ & $28,4 \pm 0,7^{\mathrm{b}}$ & $1,3 \pm 0,1$ & $289,5 \pm 27,5$ & $827,3 \pm 11,4$ & $-10,0$ \\
\hline $\begin{array}{l}\text { PA6/ABS/Cop. } \\
(10,0 \% \mathrm{MA})^{\mathrm{e}}\end{array}$ & $30,7 \pm 0,8^{b}$ & $1,4 \pm 0,1$ & $355,4 \pm 32,1$ & $878,4 \pm 7,4$ & $-2,5$ \\
\hline $\begin{array}{l}\text { PA6/ABS/Cop. } \\
(20,0 \% \mathrm{MA})^{\mathrm{e}}\end{array}$ & $29,2 \pm 0,3^{b}$ & $1,4 \pm 0,1$ & $419,9 \pm 40,1$ & $100,1 \pm 7,1$ & 28,0 \\
\hline
\end{tabular}

${ }^{a}$ Valores calculados com inclinação zero.

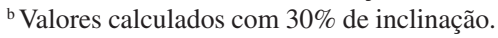

c PA6/ABS/MMA-MA - 66,5/28,5/5\%.

${ }^{\mathrm{D}} \mathrm{PA} 6 / \mathrm{ABS} / \mathrm{MMA}-\mathrm{MA}-68,25 / 29,25 / 2,5 \%$

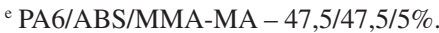

· $\mathrm{T}_{\mathrm{D}-\mathrm{F}}$ - Temperatura de transição dúctil-frágil que é o ponto médio, entre os pontos máximo e mínimo, na curva de resistência ao impacto Izod vs. temperatura 
tência ao impacto em função da temperatura, para as blendas compatibi-lizadas com o copolímero MMA-GMA. Observase que as temperaturas de transição dúctil-frágil $\left(\mathrm{T}_{\mathrm{D}-\mathrm{F}}\right)$ para todas as sequiências se mantém na faixa de $55^{\circ} \mathrm{C}$, Tabela 2 , comparadas à $\mathrm{T}_{\mathrm{D}-\mathrm{F}}$ da PA6 $\left(\sim 70^{\circ} \mathrm{C}\right)$. Ou seja, a adição de $\mathrm{ABS}$ e de compatibilizante à base de GMA e a sequiência de mistura não melhoraram significativamente a resistência ao impacto da blenda nem na temperatura ambiente e nem abaixo desta. Somente acima da $\mathrm{T}_{\mathrm{D}-\mathrm{f}}$, essa blenda apresenta-se supertenaz. Fica evidenciado que esse copolímero tem função compatibilizante na blenda PA6/ABS na temperatura ambiente, mas não pode ser comparado aos valores obtidos por Kudva $^{[17]}$ e outros ${ }^{[12]}$. Entretanto, a blenda na temperatura ambiente pode ser dita tenaz. O efeito da temperatura no comportamento de impacto é de grande importância para muitas aplicações dos termoplásticos de engenharia, que necessitam de grande ductilidade em temperaturas subambiente ${ }^{[5]}$. Esse fato pode ser comprovado, através da caracterização morfológica, que mostrará a má distribuição das partículas de borracha nestas blendas, o que, sem dúvida, é um fator responsável pelas fracas propriedades mecânicas ${ }^{[1,23,24]}$.

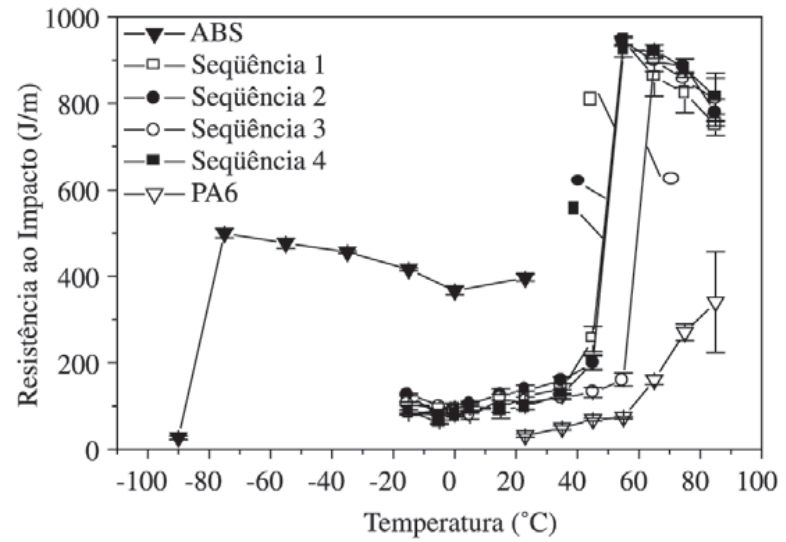

Figura 5. Efeito da temperatura na resistência ao impacto Izod da blenda PA6/ABS (70/30\%) com 5\% de compatibilizante. Sequiência 1 - PA6/MMA GMA + ABS; Seqüência 2 - ABS/MMA- GMA + PA6; Seqüência 3 - MMAGMA + PA6/ABS; Seqüência 4 - PA6/MMA-GMA + ABS/MMA-GMA.

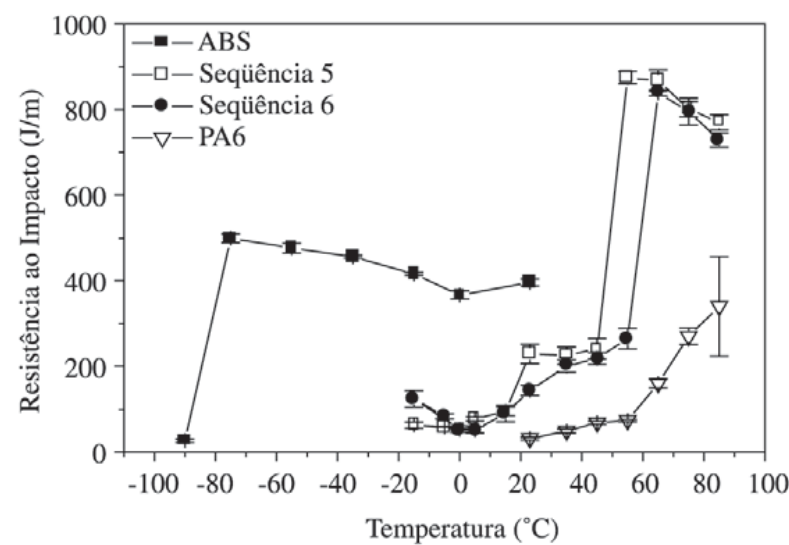

Figura 6. Efeito da temperatura na resistência ao impacto Izod da blenda PA6/ABS (50/50\%) com 5\% de compatibilizante. Seqüência 5 - PA6/ABS/ MMA-GMA; Sequiência 6 - ABS/MMA-GMA + PA6.
O efeito da temperatura na resistência ao impacto de blendas compatibilizadas com copolímero à base de anidrido maléico pode ser observado nas Figuras 7 e 8 . Nas blendas de PA6/ABS (50/50\%) com 5\% de copolímero, Figura 7 e Tabela 3, observa-se que, quando o conteúdo de MA no copolímero diminui (20 para 3\% MA), a temperatura de transição dúctil-frágil $\left(\mathrm{T}_{\mathrm{D}-\mathrm{F}}\right)$ diminui $\left(28\right.$ para $\left.-10{ }^{\circ} \mathrm{C}\right)$. A blenda sem compatibilizante, entretanto, é frágil sobre toda a faixa de temperatura analisada. A blenda contendo 3, 5 e 10\%MA no copolímero é supertenaz (acima de $800 \mathrm{~J} / \mathrm{m}$ ) na temperatura ambiente, mas somente as que contêm 3 e $5 \%$ MA são as que permanecem tenazes quando a temperatura cai abaixo de zero. Tais resultados são comparáveis aos obtidos por Majumdar et al. ${ }^{[11]}$, que usaram um ABS e uma PA6 semelhantes aos usados neste trabalho. Resultados parecidos foram observados para o sistema PBT/ABS ${ }^{[25]}$. Foi argumentado que quantidades moderadas de funcionalidade GMA no compatibilizante e pequenas quantidades de compatibilizante na blenda são suficientes para reduzir significativamente a $\mathrm{T}_{\mathrm{D}-\mathrm{F}}$ e melhorar a dispersão do $\mathrm{ABS}$, enquanto que quantidades mais altas de funcionalidade GMA

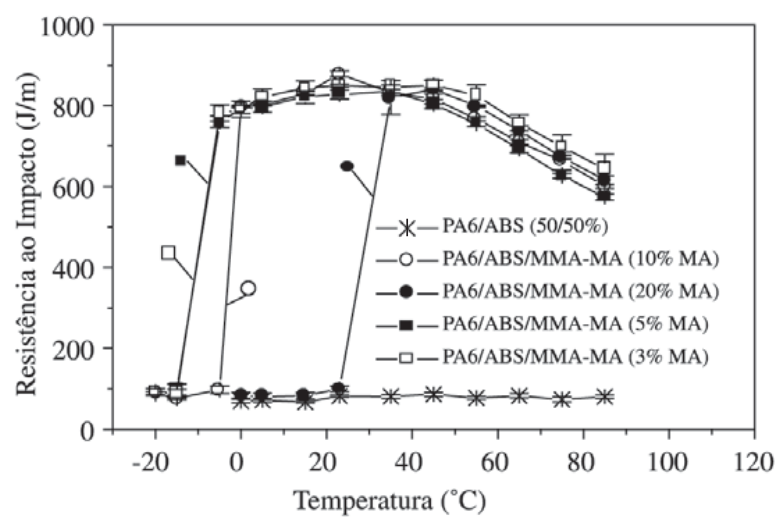

Figura 7. Efeito da temperatura na resistência ao impacto Izod da blenda binária PA6/ABS (50/50\%) e ternária PA6/ABS/MMA-MA (47,5/47,5/ $5 \%$ ) contendo quantidades variadas de MA

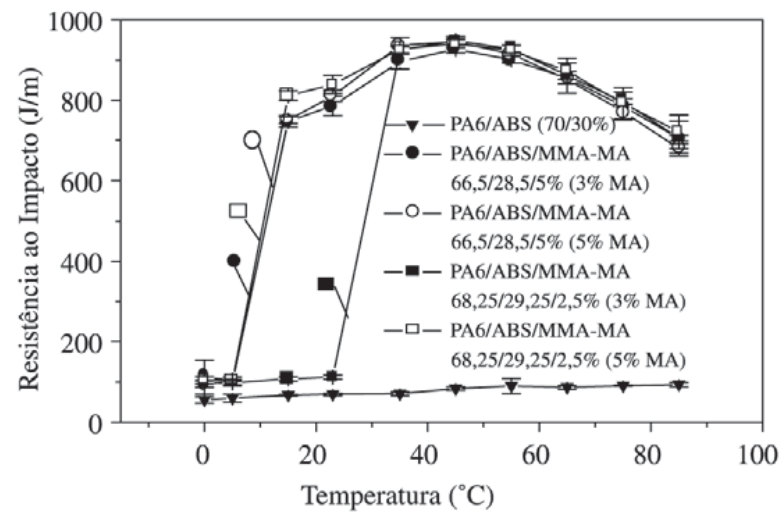

Figura 8. Efeito da temperatura na resistência ao impacto Izod da blenda binária PA6/ABS (70/30\%) e ternárias PA6/ABS/MMA-MA (66,5/28,5/ $5 \%$ e $68,25 / 29,25 / 2,5 \%$ ) contendo 3 e $5 \%$ MA. 
aumentaram a resistência ao impacto na temperatura ambiente mas não melhoraram suficientemente esta propriedade em baixas temperaturas e, além disso, elevam a viscosidade da blenda, o que é indesejável para algumas técnicas de processamento. A Figura 8 apresenta a curvas de resistência ao impacto em função da temperatura das blendas PA6/ABS, para a composição 70/30 \%. A blenda sem compatibilizante é frágil em toda a faixa de temperatura analisada. Com $5 \%$ de compatibi-lizante, elas são tenazes $(\sim 800 \mathrm{~J} / \mathrm{m})$ na temperatura ambiente e permanecem assim até $15{ }^{\circ} \mathrm{C}$, conforme mostra também a Tabela 3. Já com 2,5\% de compatibilizante, ela é tenaz na temperatura ambiente, quando o conteúdo de anidrido maléico no compatibilizante é 5\%. Para valor inferior, neste caso $3 \%$, ela é frágil na temperatura ambiente. Além disso, a temperatura de transição dúctil-frágil é maior comparada com as blendas anteriores. Esses resultados são provavelmente devidos à maior quantidade de PA6 na blenda, que é frágil em temperatura ambiente e só se torna dúctil quando a temperatura está acima de sua $\mathrm{T}_{\mathrm{g}}$. Portanto, uma maior concentração de PA6 desloca a temperatura de transição para valores maiores. Fica evidenciado, também, que o conteúdo de anidrido maléico afeta a temperatura de transição dúctil-frágil $\left(\mathrm{T}_{\mathrm{D}-\mathrm{F}}\right)$ e que existe um conteúdo ótimo de MA, abaixo do qual, a blenda é frágil em temperaturas ambiente e subambiente, e que o copolímero MMA-MA pode ser utilizado como compatibilizante das blendas PA6/ABS.

\section{Conclusões}

O efeito dos copolímeros MMA-GMA e MMA-MA como compatibilizantes das blendas PA6/ABS foi investigado. A blenda polimérica PA6/ABS pode ser considerada frágil e incompatível, devido às interações moleculares desfavoráveis entre os componentes. A blenda compatibilizada com MMA-GMA apresentou-se dúctil na temperatura ambiente e frágil em temperaturas logo abaixo desta, dependendo da sequiência de mistura que, como observado, influencia nas propriedades do material. Já a blenda PA6/ABS/MMA-MA apresentou excelente desempenho sob impacto, evidenciando a presença efetiva do copolímero como agente de compatibilização reativo deste sistema. $\mathrm{O}$ aumento do conteúdo de MA no copolímero não parece ter efeito significante na blenda, ou seja, baixos conteúdos de MA (3\%), para a composição 50/50\%, são suficientes para a tenacifi-cação da PA6. A resistência ao impacto atingiu valores acima de $800 \mathrm{~J} / \mathrm{m}$. O copolímero MMA-MA foi capaz de manter um balanço de propriedades, isto é, rigidez pouco alterada da matriz e aumento significativo sob impacto.

\section{Agradecimentos}

Os autores agradecem à Basf pelo fornecimento da poliamida 6 , à Bayer pelo fornecimento do $\mathrm{ABS}$ e à Nitriflex pelo uso de seus laboratórios para a caracterização do mesmo, ao PRONEX (Núcleo de Reologia e Processamento de Polímeros - DEMa/UFSCar) e à FAPESP pelo apoio financeiro.

\section{Referências Bibliográficas}

1.Araújo, E.M. Tenacificação da poliamida 6 com ABS por meio da técnica de compatibilização in situ com o uso de copolímeros acrílicos reativos, Tese de Doutorado, Universidade Federal de São Carlos, Brasil, 2001.

2. Brydson, J.A. Plastics Materials, Newnes-Butterworths, London, 1975.

3. Wu, C.-J.; Kuo, J.-F.; Chen, C.-Y. Polym. Engng. Sci., 33, p. $1329,1993$.

4. Triacca, V.J.; Ziaee, S.; Barlow, J.W.; Keskkula, H.; Paul, D.R. Polymer, 32, p. 1401, 1991.

5. Hage Jr., E.; Hale, W.; Keskkula, H.; Paul, D.R. Polymer, 38, p. 3237, 1997.

6. Jang, S.P.; Kim, D. Polym. Engng. Sci., 40, p. 1635, 2000.

7. Lee, Y.; Char, K. Macromolecules, 27, p. 2603, 1994.

8. Misra, A.; Sawhney, G.; Kumar, R.A. J. Ap. Pol. Sci., 50, p.1179, 1993.

9. Majumdar, B.; Keskkula, H.; Paul, D.R. Polym. Prepr., 35, p. 850, 1994.

10. Majumdar, B.; Keskkula, H.; Paul, D.R. J. Pol. Sci.: part B: pol. phys., 32, p. 2127, 1994.

11. Majumdar, B.; Keskkula, H.; Paul, D.R. Polymer, 35, p. 5453, 1994.

12. Majumdar, B.; Keskkula, H.; Paul, D.R. Polymer, 35, p. 5468, 1994.

13. Carrot, C.; Guillet, J.; May, J.F. Plast. Rubber Comp. Proc. Appl., 16, p. 61, 1991.

14. Majumdar, B.; Keskkula, H.; Paul, D.R. J. App. Pol. Sci., 54, p. 339, 1994.

15. Kim, B.K.; Lee, Y.M.; Jeong, H.M. Polymer, 34, p. 2075, 1993.

16. Majumdar, B.; Keskkula, H.; Paul, D.R. Polymer, 35, p. 3164, 1994.

17. Kudva, R.A.; Keskkula, H.; Paul, D.R. Polymer, 39, p. 2447, 1998.

18. Aoki, Y.; Watanabe, M. Pol. Eng. Sci., 32, p. 878, 1992.

19. Howe, D.V.; Wolkowicz, M.D. Pol. Eng. Sci., 27, p. 1582, 1987.

20. Hale, W.; Keskkula, H.; Paul, D.R. Polymer, 40, p. 365, 1999.

21. Oswald, T.A.; Menges, G. Materials Science of Polymers for Engineers, Hanser Publishers, New York, 1996.

22. Oshinski, A.J.; Keskkula, H.; Paul, D.R. Polymer, 37, p. 4919, 1996.

23. Araújo, E.M.; Hage Jr., E.; Carvalho, A.J.F. J. App. Pol. Sci., 87, p. 842, 2003.

24. Araújo, E.M.; Hage Jr., E.; Carvalho, A.J.F. J. App. Pol. Sci. (in press).

25. Hale, W.R.; Pessan, L.A.; Keskkula, H.; Paul, D.R. Polymer, 40, p. 4237, 1999.

Recebido: 22/01/2003

Aprovado: 07/07/03 